\title{
Solitary plasma rings and magnetic field generation involving gravity and differential rotation
}

\begin{abstract}
B. Coppi
Consorzio Interuniversitario per la Fisica dello Spazio, 10133 Torino, Italy

e-mail: coppi@mit.edu

Received 14 October 2011 / Accepted 4 October 2012

ABSTRACT

A new theoretical framework for describing how magnetic fields are generated and amplified is provided by finding magnetogravitational modes that involve gravity, density gradients, and differential rotation in an essential way. Other factors, such as the presence of a high temperature particle population or of a temperature gradient, can contribute to their excitation. These modes identified by a linearized analysis are shown to be important for the evolution of plasma disks surrounding black holes toward different configurations. Since the nonlinear development of these modes can lead to radially localized regions with a relatively small differential rotation, new stationary structures have been identified, in the (fully) nonlinear limit, which are localized radially over regions with negligible gradients of the rotation frequency. These structures, characterized by solitary plasma rings, do not involve a pre-existing "seed" magnetic field, unlike other configurations found previously. The relevant magnetic energy density is comparable to the gravitationally confined plasma pressure. The "source" of these configurations is the combination of the gravitational force and of the plasma density gradient orthogonal to it that is an important factor in the theory of magneto-gravitational modes, another important factor being an anisotropy of the plasma pressure.
\end{abstract}

Key words. plasmas - magnetohydrodynamics (MHD) - instabilities - black hole physics

\section{Introduction}

The investigations undertaken with the purpose of identifying the plasma and field configurations around black holes have led to envisioning a clear sequence of processes and a theoretical framework for generating and amplifying significant magnetic fields in the Universe. The relevant physical factors for these processes are both the plasma differential rotation and the (vector) product of the gravitational force and of the orthognal density gradient. In particular, starting from a "conventional" disk surrounding a compact object in which a "seed" magnetic field is present, magneto-gravitational modes, which can be excited and produce a rapid amplification of the field, are found. These modes, which are described by a linearized theory, can be axisymmetric or of the trailing spiral type with low toroidal mode numbers.

In particular, by adopting a fully nonlinear approach at first, stationary axisymmetric configurations are identified that can be regarded as the result of the nonlinear evolution of magnetogravitational modes. In particular, considering a region in which the rotation frequency gradient is small when compared to the Keplerian rotation frequency, solitary ring configurations are found that do not depend on the presence of a seed magnetic field. In fact, the local "flattening" of the rotation frequency can be envisioned to result from the excitation of a magnetogravitational mode as the "driving factor" of the identified nonlinear configurations is the (vector) product of the gravitational force and of the density gradient orthogonal to it. This analysis is followed by the complete linearized theory of the magnetogravitational modes emerging from a conventional disk configuration for which an important additional driving factor is the differential rotation of Keplerian orbits.
In this connection we mention that a large category of black holes can be referred to as "shining" in view of the high-energy radiation emission that can be associated with them. This emission is attributed to accretion of high-energy plasmas that surround these objects. However, this generic interpretation leads to opening a number of questions concerning both (i) the specific processes that can allow accretion to occur, or can prevent it as in the case of "dark" black holes, and (ii) the nature of the plasmas that can be involved. In fact, a fairly complete theoretical description of these plasmas would require consideration of the full phase space. A relevant observation is that X-ray astronomy has shown that these plasmas can access characteristic "states", on the basis of their radiation emission, which are nonthermal and correspond to non-Maxwellian distributions of the particle populations that are responsible for the emission. For the sake of simplicity, the analysis that is presented here adopts a single fluid description with an isotropic pressure tensor. In fact, the theory of nonlinear stationary configurations (e.g. solitary ring structures) and of the related magneto-gravitational modes has been developed (Coppi 2012) for plasmas with two components, one of which has a higher longitudinal (to the magnetic field) pressure than that of the population with isotropic pressure. This theory will be the subject of a subsequent paper.

The present paper is organized as follows. In Sect. 2, the basic assumptions that are made in order to find nonlinear, stationary, and axisymmetric configurations of plasmas and magnetic fields are described. In Sect. 3 the master equation is derived from the total momentum conservation equation by assuming a scalar plasma pressure and relates the plasma density distribution to a consistent magnetic field configuration. In Sect. 4 two classes of radially localized configurations are introduced and the master equation for the locally differential rotator 
configurations is given. In Sect. 5 the main features of locally rigid rotor configurations are illustrated, the relevant form of the master equation is discussed, and the important fact is pointed out that a seed magnetic field does not appear in the theory for these configurations. In Sect. 6 the vertical momentum density balance equation is introduced. This relates the plasma pressure to the magnetic field configuration and is to be analyzed in combination with the master equation. In Sect. 7 a class of special solutions for the nonlinear equations introduced in earlier sections that are separable functions of the vertical and radial coordinates is identified. In Sect. 8 a solitary ring solution is found in the context of the locally differential rotor class of configurations. In Sect. 9 the periodic sequence of rings for the same class of configurations, and published earlier, is recovered. In Sect. 10 the master equation of the locally rigid rotor class of configurations is analyzed. In Sects. 11 and 12 a periodic ring sequence, as well as a solitary ring solution, are found for the same class of configurations. In Sect. 13 the basic equation describing the magneto-gravitational modes in the linearized approximation is introduced. In Sect. 14 the spatial profiles and the growth rates of two different kinds of magneto-gravitational modes are analyzed. Section 15 describes the tri-dimensional spiral configurations, which are of the ring type and were found earlier in the linearized approximation. Then nonlinear ring configurations with a corresponding spiral structure are identified. In Sect. 16 a theoretical framework is proposed in order to explain the generation of significant magnetic fields in the Universe by the combined effects of gravity, density gradient, differential rotation, and other factors. The excitation of magneto-gravitational modes and the formation of locally rigid rotation configurations have the key role in this. In Sect. 17 final consideration is given to both the limitations of the presented theory and its envisioned developments.

\section{Basic assumptions}

Given the intent to identify the simplest stationary plasma and field configurations that can (theoretically) exist around compact collapsed objects such as black holes, we limit our analysis at first to axisymmetric geometries with the following characteristics:

a) Perfectly conducting plasma conditions are considered and, consequently,

$\boldsymbol{V}=\alpha_{\mathrm{v}} \boldsymbol{B}+\Omega(\psi) \boldsymbol{R} \boldsymbol{e}_{\varphi}$

where $\boldsymbol{V}$ is the plasma flow velocity, $\psi=\psi(R, z)$ is the magnetic surface function, and cylindrical coordinates are used.

b) No appreciable poloidal flow velocity is included.

c) The relevant particle distributions in velocity space are close to Maxwellian and refer to a scalar pressure $(\boldsymbol{P}=p \boldsymbol{I})$ is appropriate.

d) The relevant Lorentz force does not have a toroidal component and, consequently, the corresponding magnetic field configurations are represented by

$\boldsymbol{B}=\frac{1}{R}\left[\nabla \psi \times \boldsymbol{e}_{\varphi}+I(\psi) \boldsymbol{e}_{\varphi}\right]$,

as, in this case, the Lorenz force $\boldsymbol{F}_{\mathrm{L}}$ is given simply by

$\boldsymbol{F}_{\mathrm{L}}=\frac{1}{4 \pi R^{2}}\left(\Delta_{*} \psi+I \frac{\mathrm{d} I}{\mathrm{~d} \psi}\right) \nabla \psi$,

where

$\Delta_{*} \psi \equiv \frac{\partial^{2} \psi}{\partial z^{2}}+R \frac{\partial}{\partial R}\left(\frac{1}{R} \frac{\partial}{\partial R} \psi\right)$, e) A Newtonian gravitational potential $\Phi_{G}$ is included for simplicity. In particular, for the relative thin plasma structures that we analyze,

$\nabla \Phi_{\mathrm{G}} \simeq-\frac{V_{\mathrm{K}}^{2}}{R}\left(\boldsymbol{e}_{R}+\frac{z}{R} \boldsymbol{e}_{Z}\right)$

where

$V_{\mathrm{K}}^{2} \equiv \frac{G M_{*}}{R} \equiv \Omega_{\mathrm{K}}^{2} R^{2}$,

and $\Omega_{\mathrm{K}}$ is the Keplerian frequency. We observe that when considering scale distances that are relatively close to black holes, we have found it convenient to make use of effective gravitational potentials in order to include relevant General Relativity effects in the theory (Coppi 2011).

\section{Relevant form of the master equation}

Referring to the total momentum conservation

$-\rho\left(\nabla \Phi_{\mathrm{G}}+\Omega^{2} \boldsymbol{R e}_{R}\right)=-\nabla p+\frac{1}{c} \boldsymbol{J} \times \boldsymbol{B}$,

we observe that

$$
\begin{aligned}
\nabla \times\left(\rho \nabla \Phi_{\mathrm{G}}+\rho \Omega^{2} R \boldsymbol{e}_{R}\right)= \\
\boldsymbol{e}_{\phi}\left\{\frac{\partial \rho}{\partial z}\left(R \Omega^{2}+\frac{\partial \Phi_{\mathrm{G}}}{\partial R}\right)+\rho R 2 \Omega \frac{\partial \Omega}{\partial z}-\frac{\partial \rho}{\partial R} \frac{\partial \Phi_{\mathrm{G}}}{\partial z}\right\}
\end{aligned}
$$

and

$$
\begin{aligned}
& \nabla \times\left(\frac{1}{c} \boldsymbol{J} \times \boldsymbol{B}\right)=\frac{1}{4 \pi} \nabla \times(\boldsymbol{B} \cdot \nabla \boldsymbol{B})= \\
& \frac{1}{4 \pi R^{2}}\left[-\frac{2}{R}\left(\Delta_{*} \psi+I \frac{\mathrm{d} I}{\mathrm{~d} \psi}\right) \boldsymbol{e}_{R}+\nabla\left(\Delta_{*} \psi\right)\right] \times \nabla \psi .
\end{aligned}
$$

Therefore, we obtain the "master equation" (Coppi 2011) that relates $\psi$ to $\rho$

$R \frac{\partial}{\partial z}\left(\Omega^{2} \rho\right)+\frac{\partial \rho}{\partial z} \frac{\partial \Phi_{\mathrm{G}}}{\partial R}-\frac{\partial \rho}{\partial R} \frac{\partial \Phi_{\mathrm{G}}}{\partial z} \simeq \frac{1}{4 \pi R^{2}}$

$\times\left\{\left[\frac{2}{R}\left(\Delta_{*} \psi+I \frac{\mathrm{d} I}{\mathrm{~d} \psi}\right)-\frac{\partial}{\partial R}\left(\Delta_{*} \psi\right)\right] \frac{\partial \psi}{\partial z}+\left[\frac{\partial}{\partial z}\left(\Delta_{*} \psi\right)\right] \frac{\partial \psi}{\partial R}\right\}$

We consider local plasma and field structures in an interval $\left|R-R_{0}\right|<R_{0}$ around $R-R_{0}$, and we indicate the characteristic scale distance, over which $R-R_{0}$ and $z$ vary, by $\Delta_{R}$ and $\Delta_{z}$, respectively. Here $\rho=\rho\left(R_{*}, \bar{z}^{2}\right), \psi=\psi\left(R_{*}, \bar{z}^{2}\right)$ for

$R_{*} \equiv \frac{R-R_{0}}{\Delta_{R}}, \bar{z} \equiv \frac{z}{\Delta_{z}}$ and $\Delta_{R}^{2} \lesssim \Delta_{z}^{2} \ll R_{0}^{2}$.

In particular, we consider $\rho$ to be an even function of both $R_{*}$ and $\bar{z}$ that is positive for all values of $R_{*}$ and $\bar{z}$.

\section{Classes of local configurations}

Now we consider two classes of local $\left(\left(R-R_{0}\right)^{2} \ll R_{0}^{2}\right)$ configurations

a) locally differential rotator configurations;

b) locally rigid rotator configurations.

In the first case we assume that a seed magnetic field $B_{0} \boldsymbol{e}_{z}$ is present corresponding to a magnetic surface function 
$\psi_{0} \simeq B_{0} R_{0} R$ and referring to a surface function $\psi \simeq \psi_{0}+\psi_{1}$ with $\left|\psi_{1}\right|<\psi_{0}$, but $B_{z} \sim\left|\psi_{1}\right| /\left(\Delta_{R} R_{0}\right)>B_{0} \sim \psi_{0} / R_{0}^{2}$. Then we take $\alpha_{v}=0$ and

$$
\begin{aligned}
v_{\phi} \simeq & \Omega(\psi) R \simeq \Omega_{\mathrm{K}}\left(R_{0}\right) R_{0}+\Omega_{\mathrm{K}}\left(R_{0}\right)\left(R-R_{0}\right) \\
& +\left(\frac{\mathrm{d} \Omega_{\mathrm{K}}}{\mathrm{d} R}\right)_{R=R_{0}} R_{0}\left[\left(R-R_{0}\right)+\psi_{1} /\left(\mathrm{d} \psi_{0} / \mathrm{d} R\right)\right]
\end{aligned}
$$

and define

$\delta \Omega=\left[\frac{\mathrm{d} \Omega_{\mathrm{K}}}{\mathrm{d} R} / \frac{\mathrm{d} \psi_{0}}{\mathrm{~d} R}\right]_{R=R_{0}} \psi_{1}$.

In this case considering the asymptotic limit where $R_{0} \Delta_{R}>\Delta_{z}^{2}>$ $\Delta_{R}^{2}$ the master equation reduces to

$$
\begin{aligned}
-\Omega_{\mathrm{D}}^{2} R_{0} \frac{\partial}{\partial z}\left(\rho \frac{\psi_{1}}{\psi_{0}}\right) \simeq & \frac{1}{4 \pi R_{0}^{2}}\left\{\left[\left(\frac{\partial^{3}}{\partial z^{3}} \psi_{1}\right) \frac{\partial \psi_{1}}{\partial R}-\left(\frac{\partial^{3}}{\partial R^{3}} \psi_{1}\right) \frac{\partial \psi_{1}}{\partial z}\right]\right. \\
& \left.+\left[\left(\frac{\partial}{\partial z} \frac{\partial^{2}}{\partial R^{2}} \psi_{1}\right) \frac{\partial \psi_{1}}{\partial R}-\left(\frac{\partial}{\partial R} \frac{\partial^{2}}{\partial z^{2}} \psi_{1}\right) \frac{\partial \psi_{1}}{\partial z}\right]\right\}
\end{aligned}
$$

where

$\Omega_{\mathrm{D}}^{2}=-R_{0} \frac{\mathrm{d}}{\mathrm{d} R} \Omega_{\mathrm{K}}^{2}=3 \Omega_{0}^{2}$.

Clearly the symmetries of this equation indicate that $\delta \Omega$ and therefore $\psi_{1}$ have to be odd functions of $R_{*}$. We observe that $\rho \Omega_{\mathrm{D}}^{2}$ is the driving factor for the field configuration represented by $\psi_{1}$. Then we note that the scale distance $\Delta_{z}$ does not affect Eq. (4.2) in the limit where $\Delta_{R}^{2} / \Delta_{z}^{2}$ can be neglected, and in this case, it was analyzed earlier (Coppi \& Rousseau 2006):

$$
\frac{\Delta_{R}}{R_{0}} \sim\left(\frac{\psi_{1} \psi_{0}}{4 \pi \rho R_{0}^{6} \Omega_{\mathrm{D}}^{2}}\right)^{1 / 3} .
$$

\section{Locally rigid rotor configurations}

The locally rigid rotor configurations we consider are characterized by

$V_{\phi}=\Omega_{0} R$, where $\Omega_{0} \equiv \Omega_{\mathrm{K}}\left(R=R_{0}\right)$,

then

$\Omega_{0} R=\alpha_{V} B_{\phi}+\Omega(\psi) R$.

We observe that an important property of this class of configurations is that the presence of a seed magnetic field is not required.

Clearly, the simplest case to analyze is that where $B_{\phi}=0$ and $\Omega(\psi)=\Omega_{0}$. Then the master Eq. (3.4) reduces to

$$
\begin{aligned}
\Omega_{0}^{2}\left[z \frac{\partial \rho}{\partial R}-3\left(R-R_{0}\right) \frac{\partial \rho}{\partial z}\right] \simeq \frac{1}{4 \pi R_{0}^{2}}\left\{\left[\left(\frac{\partial^{3}}{\partial z^{3}} \psi\right) \frac{\partial \psi}{\partial R}-\left(\frac{\partial^{3}}{\partial R^{3}} \psi\right) \frac{\partial \psi}{\partial z}\right]\right. \\
\left.+\left[\left(\frac{\partial}{\partial z} \frac{\partial^{2}}{\partial R^{2}} \psi\right) \frac{\partial \psi}{\partial R}-\left(\frac{\partial}{\partial R} \frac{\partial^{2}}{\partial z^{2}} \psi\right) \frac{\partial \psi}{\partial z}\right]\right\} \cdot
\end{aligned}
$$

As we can see from Eq. (5.3),

$\Omega_{0}^{2} \frac{\Delta_{z}}{\Delta_{R}} \rho \sim \frac{1}{4 \pi R_{0}^{2}} \frac{\psi_{1}^{2}}{\Delta_{R}^{3}} \frac{1}{\Delta_{z}}$, which corresponds to

$\Omega_{0}^{2} \Delta_{z}^{2} \sim \frac{B_{z}^{2}}{4 \pi \rho}$

If we consider that $\Delta_{z}$ could be comparable to $\Delta_{\mathrm{G}} \sim v_{\text {thi }} / \Omega_{\mathrm{K}}$, the height of a conventional thermal disk (see Sect. 6), then the magnetic energy density can be of the same order of magnitude as the plasma thermal energy density. The plasma pressures corresponding to the solution for $\rho$ and $\psi$ given by Eqs. (4.2) and (5.3) are obtained by the vertical momentum density conservation analyzed in Sect. 6.

We note that in this limit $\psi$ can be either an odd or an even function of $R_{*}$ and an even or odd function of $z$. Clearly the driving factor of the relevant magnetic field configurations is the term $\left(\nabla \rho \times \nabla \Phi_{\mathrm{G}}\right) \cdot \boldsymbol{e}_{\varphi}$, which is a combination of the gravitational force and the density gradient orthogonal to it.

\section{Vertical momentum density conservation equation}

The vertical equilibrium equation connects the total plasma pressure to the particle density and the magnetic field configuration. In particular, this equation can be written as

$0 \simeq-\Omega_{\mathrm{K}}^{2} \rho z-\frac{\partial}{\partial z}\left(p+\frac{B^{2}}{8 \pi}\right)+\frac{1}{4 \pi}(\boldsymbol{B} \cdot \nabla \boldsymbol{B})_{z}$,

and we find it convenient to separate $p$ into $p_{\mathrm{G}}+p_{\mathrm{M}}$ where

$2 \frac{\partial}{\partial z^{2}} p_{\mathrm{G}}=-\Omega_{\mathrm{K}}^{2} \rho$

Thus we may define the temperature

$T_{\mathrm{G}}=\frac{m_{i}}{2} \frac{p_{\mathrm{G}}}{\rho}$

where $m_{i}$ is the mass of the nuclei of which the plasma is composed and consider sufficiently high values of $T_{\mathrm{G}}$ such that $p=p_{\mathrm{G}}+p_{\mathrm{M}}$ is always positive. A brief discussion of the profiles $T_{\mathrm{G}}=T_{\mathrm{G}}\left(z^{2}\right)$ that can be present in a "conventional" disk is given in the appendix.

Clearly,

$\frac{\partial p_{\mathrm{M}}}{\partial z}=-\frac{1}{8 \pi} \frac{\partial}{\partial z}\left(B_{R}^{2}+B_{\phi}^{2}\right)-\frac{1}{4 \pi}\left(B_{R} \frac{\partial}{\partial R} B_{z}\right)$.

If we consider axisymmetric configurations for which $I=I(\psi)$, this reduces to

$\frac{\partial p_{\mathrm{M}}}{\partial z}=-\frac{1}{4 \pi R^{2}} \frac{\partial \psi}{\partial z}\left(\Delta_{*} \psi+I \frac{\mathrm{d} I}{\mathrm{~d} \psi}\right)$

Moreover if we assume that $p_{\mathrm{M}}=p_{\mathrm{M}}(\psi), \partial p_{\mathrm{M}} / \partial_{z}=$ $\left(\mathrm{d} p_{\mathrm{M}} / \mathrm{d} \psi\right) \partial \psi / \partial z$ and we obtain the so-called "G-S equation" for magnetically confined laboratory plasmas. In fact, the socalled "pulsar equation" describing the magnetic configuration of an axisymmetric plasma surrounding a rotating neutron star was derived first and solved along similar lines by Cohen et al. (1973).

In the present case we do not consider $p_{\mathrm{M}}=p_{\mathrm{M}}(\psi)$, and have, for configurations localized around $R=R_{0}$,

$\frac{\partial}{\partial z}\left\{p_{\mathrm{M}}+\frac{1}{8 \pi R_{0}^{2}}\left[I^{2}+\left(\frac{\partial \psi}{\partial z}\right)^{2}\right]\right\} \simeq-\frac{1}{4 \pi R_{0}^{2}} \frac{\partial \psi}{\partial z} \frac{\partial^{2} \psi}{\partial R^{2}}$ 
In particular, if we introduce the dimensionless variables $R_{*}$ and $\bar{z}$, Eq. (6.6) becomes

$\frac{\partial}{\partial \bar{z}}\left\{p_{\mathrm{M}}+\frac{1}{8 \pi R_{0}^{2}}\left[I^{2}+\frac{1}{\Delta_{z}^{2}}\left(\frac{\partial \psi}{\partial \bar{z}}\right)^{2}\right]\right\} \simeq-\frac{1}{4 \pi R_{0}^{2} \Delta_{R}^{2}} \frac{\partial \psi}{\partial \bar{z}} \frac{\partial^{2} \psi}{\partial R_{*}^{2}}$.

\section{Factorized solutions}

Now we limit our analysis to functions $\psi$, which can be factorized as

$\psi=\psi_{\mathrm{N}} \bar{\psi}_{*}\left(R_{*}\right) \exp \left(-\frac{\bar{z}^{2}}{2}\right)$

and Eq. (6.7) becomes

$\frac{\partial}{\partial \bar{z}}\left\{p_{\mathrm{M}}+\frac{I^{2}}{8 \pi R_{0}^{2}}\right\} \simeq \frac{1}{4 \pi R_{0}^{2}}\left\{\frac{1}{\Delta_{z}^{2}} \bar{\psi}_{*}^{2}+\frac{1}{\Delta_{R}^{2}} \bar{\psi}_{*} \frac{\mathrm{d}^{2} \bar{\psi}_{*}}{\mathrm{~d} \bar{R}_{*}^{2}}\right\} \exp \left(-\bar{z}^{2}\right)$.

Therefore

$p_{\mathrm{M}}+\frac{I^{2}}{8 \pi R_{0}^{2}} \simeq-\frac{1}{8 \pi R_{0}^{2}}\left\{\frac{1}{\Delta_{z}^{2}} \bar{\psi}_{*}^{2}+\frac{1}{\Delta_{R}^{2}} \bar{\psi}_{*} \frac{\mathrm{d}^{2} \bar{\psi}_{*}}{\mathrm{~d} R_{*}^{2}}\right\} \exp \left(-\bar{z}^{2}\right)$.

Then we refer to Eq. (6.2), assume for simplicity that $T_{\mathrm{G}}=T_{\mathrm{G} 0}=$ constant, write

$\frac{4}{m_{i}} \frac{T_{\mathrm{G} 0}}{\Omega_{\mathrm{k}}^{2}} \frac{\partial \rho}{\partial z^{2}}=-\rho$

and define

$\Delta_{\mathrm{G}}^{2} \equiv \frac{4 T_{\mathrm{G}}}{m_{i} \Omega_{\mathrm{K}}^{2}}$.

Consequently,

$\rho=\rho_{\mathrm{N}} \bar{\rho}_{*}\left(R_{*}\right) \exp \left(-\frac{z^{2}}{\Delta_{\mathrm{G}}^{2}}\right)$.

The relationship between $\Delta_{\mathrm{G}}^{2}$ and $\Delta_{z}^{2}$ will depend on the classes of solutions of the master equation that we consider. In particular, for the locally differentially rotator configurations we find

$\Delta_{\mathrm{G}}^{2}=2 \Delta_{z}^{2}$

and for the locally rigid rotor configurations

$\Delta_{\mathrm{G}}^{2}=\Delta_{z}^{2}$.

We also observe that

$\frac{\partial}{\partial z} I^{2}=2 I \frac{\partial \psi}{\partial z} \frac{\mathrm{d} I}{\mathrm{~d} \psi}$

and in the case where $\psi \simeq \psi_{0}+\psi_{1}$, we have

$\frac{\partial}{\partial z} I^{2} \simeq-\left[\frac{\mathrm{d}}{\mathrm{d} \psi_{0}} I^{2}\left(\psi_{0}\right)\right] \psi_{\mathrm{N}} \bar{\psi}_{*}\left(R_{*}\right) \frac{1}{\Delta_{z}} \exp \left(-\frac{\bar{z}^{2}}{2}\right)$.

Therefore in the present case this component of $p_{\mathrm{M}}$ has the same $z$-profile as $p_{\mathrm{G}}$.

\section{Locally differential rotator configurations: solitary rings}

A radially localized solution of the master Eq. (4.2) that involves a Gaussian function is

$\bar{\psi}_{*}=R_{*} \exp \left(-\frac{1}{2} R_{*}^{2}\right)$.

The expression for $\bar{\rho}$ that we obtain in this case is

$\bar{\rho}_{*}=2\left[\frac{\Delta_{R}^{2}}{\Delta_{z}^{2}}+R_{*}^{2}\left(1-\frac{\Delta_{R}^{2}}{\Delta_{z}^{2}}\right)\right] \exp \left(-\frac{1}{2} R_{*}^{2}\right)$.

Requiring that $\Delta_{R}^{2} / \Delta_{z}^{2} \leq 1$. We observe that the profile (9.2) corresponds to a single ring when

$\frac{2}{3}<\frac{\Delta_{R}^{2}}{\Delta_{z}^{2}} \leq 1$.

Clearly, $B_{R} \sim B_{z}$ when $\Delta_{R} \sim \Delta_{z}$. We note that, if we take $\psi_{\mathrm{N}} \sim$ $\psi_{0}$ and $v_{\mathrm{A}}^{2} \sim v_{\phi}^{0} v_{\mathrm{th}}^{0}$, we have $v_{\mathrm{A}}^{2} \sim v_{\phi} \Omega_{\mathrm{k}} \Delta_{R}$ and $v_{\mathrm{A}}^{2} \sim v_{\phi} v_{\mathrm{th}}^{0}$ if $\Delta_{R} \sim \Delta_{z}$, for $v_{\phi}=\Omega_{\mathrm{k}} R_{0}$ and $v_{\mathrm{th}}^{0} \sim\left(2 T_{\mathrm{G}}^{0} / m_{i}\right)^{1 / 2}$.

Thus we may argue that the pair of rings collapses into one ring as $\left(\Delta_{R} / \Delta_{z}\right)^{2}$ is increased. The poloidal magnetic field components now are

$B_{z} \simeq \frac{\psi_{\mathrm{N}}}{R_{0} \Delta_{R}}\left(1+R_{*}^{2}\right) \exp \left[-\frac{1}{2}\left(R_{*}^{2}+\bar{z}^{2}\right)\right]$,

and

$B_{R} \simeq \frac{\psi_{\mathrm{N}}}{R_{0} \Delta_{R}} \bar{z} \bar{R}_{*} \exp \left[-\frac{1}{2}\left(R_{*}^{2}+\bar{z}^{2}\right)\right]$,

thus the relevant magnetic surfaces exhibit two O-points, at $R_{*}=$ \pm 1 and $\bar{z}=0$. The current density $J_{\phi}$ becomes

$J_{\phi} \simeq \frac{c \psi_{\mathrm{N}}^{0}}{4 \pi R_{0}} \exp \left[-\frac{1}{2}\left(R_{*}^{2}+\bar{z}^{2}\right)\right] \times R_{*}\left[\left(1-\bar{z}^{2}\right) \frac{1}{\Delta_{z}^{2}}+\left(3-R_{*}^{2}\right) \frac{1}{\Delta_{R}^{2}}\right]$

and corresponds to opposite current channels for $R_{*}>0$ and $R_{*}<0$.

Now, if we refer to Eq. (7.3) where we take $I=0$, we have

$p_{\mathrm{M}}=-\frac{R_{*}^{2} \psi_{\mathrm{N}}^{2}}{8 \pi R_{0}^{2}}\left[\frac{1}{\Delta_{z}^{2}} \bar{z}^{2}+\frac{1}{\Delta_{R}^{2}}\left(R_{*}^{2}-3\right)\right] \exp \left[-\left(R_{*}^{2}+\bar{z}^{2}\right)\right]$

which is always negative for $R^{2}{ }_{*}>3$. On the other hand, in this case

$p_{\mathrm{G}}=p_{\mathrm{NG}}\left[\frac{\Delta_{R}^{2}}{\Delta_{z}^{2}}+R_{*}^{2}\left(1-\frac{\Delta_{R}^{2}}{\Delta_{z}^{2}}\right)\right] \exp \left[-\frac{1}{2}\left(R_{*}^{2}+\bar{z}^{2}\right)\right]$.

Therefore,

$$
\begin{aligned}
p & =p_{\mathrm{G}}+p_{\mathrm{M}}=p_{\mathrm{NG}} \exp \left(-\frac{1}{2}\left(R_{*}^{2}+\bar{z}^{2}\right)\right) \\
& \times\left\{R_{*}^{2}+\frac{\Delta_{R}^{2}}{\Delta_{z}^{2}}\left(1-R_{*}^{2}\right)+\left(\frac{\psi_{\mathrm{N}}^{2}}{8 \pi R_{0}^{2} \Delta_{R}^{2} p_{\mathrm{NG}}}\right)\right. \\
& \left.\times \exp \left(-\frac{1}{2}\left(R_{*}^{2}+\bar{z}^{2}\right)\right) R_{*}^{2}\left[\left(3-R_{*}^{2}\right)-\frac{\Delta_{R}^{2}}{\Delta_{z}^{2}} \bar{z}^{2}\right]\right\} .
\end{aligned}
$$




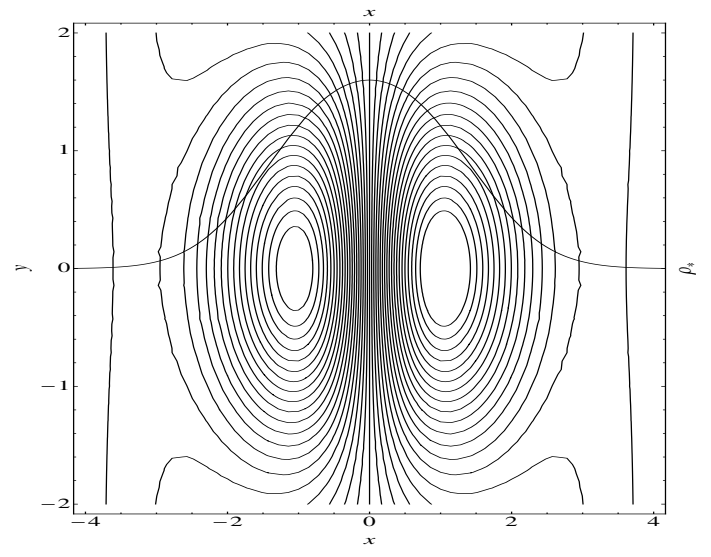

Fig. 1. Graphic representation of the magnetic surfaces for the configuration corresponding to Eq. (8.10). The curve with dotted heavy lines indicates the single ring density profiles represented by Eq. (8.2) for $\Delta_{R}^{2} / \Delta_{z}^{2}=1 / 10$.

Clearly, for adequate values of $p_{\mathrm{NG}}$ the total plasma pressure can remain positive with a finite total temperature $T$ for all values of $\bar{z}$ and $R_{*}$. We observe that the "complex" toroidal current density pattern represented by Eq. (8.6) is reminiscent of the one found for the modes identified in Coppi (2009).

We note that the complete magnetic surface function $\psi$ is, in this case,

$$
\begin{aligned}
\psi & =\psi_{1}\left(R_{*}, \bar{z}\right)+R_{*} \frac{\Delta_{R}}{R_{0}} \psi_{0} \\
& =\psi_{\mathrm{N}}\left[\bar{\psi}_{*}\left(R_{*}\right) \exp \left(-\frac{1}{2} \bar{z}^{2}\right)+R_{*} \frac{\Delta_{R}}{R_{0}} \frac{\psi_{0}}{\psi_{\mathrm{N}}}\right]
\end{aligned}
$$

and we consider $\psi_{\mathrm{N}} / \Delta_{R}>\psi_{0} / R_{0}$. Therefore, the relevant magnetic surfaces are represented by

$R_{*} \exp \left[-\frac{1}{2}\left(R_{*}^{2}+\bar{z}^{2}\right)\right]+\varepsilon_{z} R_{*}=$ const.

with $\varepsilon_{z}<1$. A graphic representation of these surfaces is given in Fig. 1.

\section{Periodic ring structures for locally differentially rotating configurations}

These structures can be found in the limit where the ratio $\Delta_{R}^{2} / \Delta_{z}^{2}$ can be considered as negligibly small. Thus they may be viewed as having a "microscopic" radial modulation, while the kind of structure identified in Sect. 8 can have a "macroscopic" radial thickness on the order of $\Delta_{z}$. In the present case case the Master Equation reduces to that derived already in (Coppi \& Rousseau 2006),

$\bar{\rho}_{*} \bar{\psi}_{*}=\frac{\mathrm{d}^{3} \bar{\psi}_{*}}{\mathrm{~d} R_{*}^{3}} \bar{\psi}_{*}-\frac{\mathrm{d}^{2} \bar{\psi}_{*}}{\mathrm{~d} R_{*}^{2}} \frac{\mathrm{d} \bar{\psi}_{*}}{\mathrm{~d} R_{*}}$,

and the considered solution is

$\bar{\psi}_{*}=\sin R_{*}+\frac{\varepsilon_{*}}{2} \sin 2 R_{*}$,

leading to the following radial density profile

$\bar{\rho}_{*}=\varepsilon_{*} \frac{\sin ^{2} R_{*}}{1+\varepsilon_{*} \cos R_{*}}$, where the dimensionless parameter $\varepsilon_{*} \leq 1 / 4$. We observe that $\bar{\psi}_{*}=\sin R_{*}$ is the solution corresponding to $\varepsilon_{*}=0$ and to a vanishing modulated density $\rho_{*}$.

Then the question that remains to be investigated is whether this periodic solution will survive when appreciable values for $\Delta_{R}^{2} / \Delta_{z}^{2}$ are considered and the assumption of separability cannot be maintained. Moreover, we observe that

$p_{\mathrm{M}} \simeq \frac{1}{8 \pi R_{0}^{2}}\left\{\frac{1}{\Delta_{R}^{2}} \psi^{2}-I^{2}\right\}$,

and given the expression (7.6) for $\rho$ if we take $I^{2} \lesssim\left(\psi / \Delta_{R}\right)^{2}$, we have no evident problems with the expression for $T_{\mathrm{M}}=$ $m_{i} p_{\mathrm{M}} /(2 \rho)$.

\section{Master equation for locally rigid rotor configurations}

Now we refer to the locally rigid rotor case and to the master Eq. (5.3). Considering the dependence of $\psi$ on $z$ indicated by Eq. (7.1), we note that in this case a more peaked expression for $\rho$ is appropriate, that is

$\rho=\rho_{\mathrm{N}} \bar{\rho}_{*}\left(R_{*}\right) \exp \left(-\bar{z}^{2}\right)$,

and that Eq. (5.3) becomes

$$
\begin{aligned}
\Omega_{0}^{2}\left[\frac{\partial \rho}{\partial R}+6 \frac{\left(R-R_{0}\right)}{\Delta_{z}^{2}} \rho\right] \simeq & \frac{1}{4 \pi R_{0}^{2} \Delta_{z}^{2}} \\
& \times\left\{\psi \frac{\partial}{\partial R}\left[\frac{\partial^{2} \psi}{\partial R^{2}}+\frac{1}{\Delta_{z}^{2}}\left(\bar{z}^{2}-1\right) \psi\right]\right. \\
& \left.-\left(\frac{\partial \psi}{\partial R}\right)\left[\frac{\partial^{2}}{\partial R^{2}}+\frac{1}{\Delta_{z}^{2}}\left(\bar{z}^{2}-1\right)\right] \psi\right\} .
\end{aligned}
$$

Then we obtain, for $\Delta_{R}^{2} / \Delta_{z}^{2} \ll 1$,

$\frac{\mathrm{d} \bar{\rho}_{*}}{\mathrm{~d} R_{*}} \simeq \frac{\mathrm{d}}{\mathrm{d} R_{*}}\left[\bar{\psi}_{*} \frac{\mathrm{d}^{2} \bar{\psi}_{*}}{\mathrm{~d} R_{*}^{2}}-\left(\frac{\mathrm{d} \bar{\psi}_{*}}{\mathrm{~d} R_{*}}\right)^{2}\right]$.

Consequently, the relationship between $\rho$ and $\psi$ can be expressed as

$\bar{\rho}_{*}=\bar{\rho}_{0}-\left(\frac{\mathrm{d} \bar{\psi}_{*}}{\mathrm{~d} R_{*}}\right)^{2}+\bar{\psi}_{*} \frac{\mathrm{d}^{2} \bar{\psi}_{*}}{\mathrm{~d} R_{*}^{2}}$.

Clearly, in the considered limit $\left(\Delta_{R}^{2} \ll \Delta_{z}^{2}\right)$,

$\frac{\partial}{\partial \bar{z}^{2}} p_{\mathrm{M}} \simeq \frac{\psi_{\mathrm{N}}^{2}}{8 \pi R_{0}^{2} \Delta_{R}^{2}} \exp \left(-\bar{z}^{2}\right) \bar{\psi}_{*}\left(R_{*}\right) \frac{\mathrm{d}^{2} \bar{\psi}_{*}}{\mathrm{~d} R_{*}^{2}}$.

Therefore,

$p_{\mathrm{M}} \simeq-\frac{\psi_{\mathrm{N}}^{2}}{8 \pi R_{0}^{2} \Delta_{R}^{2}} \bar{\psi}_{*} \frac{\mathrm{d}^{2} \bar{\psi}_{*}}{\mathrm{~d} R_{*}^{2}} \exp \left(-\bar{z}^{2}\right)$,

and if $\bar{\psi}_{*} \mathrm{~d}^{2} \psi_{*} / \mathrm{d} R_{*}^{2}<0$, we require that

$p_{\mathrm{G} 0} \bar{\rho}_{*}>\frac{\psi_{\mathrm{N}}^{2}}{8 \pi R_{0}^{2} \Delta_{R}^{2}}\left(\bar{\psi}_{*} \frac{\mathrm{d}^{2} \bar{\psi}_{*}}{\mathrm{~d} R_{*}^{2}}\right)$.

The relevant toroidal current density is about

$J_{\phi} \simeq-\frac{c}{4 \pi R_{0}}\left(\frac{1}{\Delta_{R}^{2}} \frac{\partial^{2} \psi}{\partial R_{*}^{2}}+\frac{1}{\Delta_{z}^{2}} \frac{\partial^{2} \psi}{\partial \bar{z}^{2}}\right)$. 


\section{Rigidly rotating periodic magnetic field configurations}

A particularly interesting set of field and plasma configurations that has not been looked for until now is the one for which the rotation frequencies are about constant in the region over which these configurations are radially localized. Thus we argued earlier that the development of these configurations may be considered as a candidate process for generating magnetic fields associated with the combined product of the gravitational force vertical component and of the local plasma density gradient.

In particular, we observe that the function $\sin k_{*} R_{*}$ and $\cos k_{*} R_{*}$ are solutions of Eq. (10.3) for $\bar{\rho}_{*}=0$, as in this case,

$\frac{\mathrm{d}}{\mathrm{d} R_{*}}\left[\bar{\psi}_{*} \frac{\mathrm{d}^{2} \bar{\psi}_{*}}{\mathrm{~d} R_{*}^{2}}-\left(\frac{\mathrm{d} \bar{\psi}_{*}}{\mathrm{~d} R_{*}}\right)^{2}\right]=0$.

Therefore, we may consider a periodic solution of the form

$\bar{\psi}_{*} \simeq \sin R_{*}+\frac{\alpha_{*}}{4} \sin \left(2 R_{*}\right)$,

which, given Eq. (10.4), can lead to

$\bar{\rho}_{*}=\alpha_{*}\left\{1-\frac{1}{2} \cos R_{*}\left[3-\cos ^{2} R_{*}\right]\right\}$.

\section{Rigidly rotating solitary rings}

A solitary ring solution of the master Eq. (10.2) can be found that is compatible with a realistic profile of the plasma pressure represented by $\bar{p}_{*}\left(R_{*}\right)=\bar{p}_{* \mathrm{G}}+\bar{p}_{* \mathrm{M}}\left(R_{*}\right)$. In particular we rewrite Eq. (10.2) as

$\frac{\mathrm{d} \bar{\rho}_{*}}{\mathrm{~d} R_{*}}+6 \epsilon_{0} \bar{\rho}_{*} R_{*}=\frac{\mathrm{d}}{\mathrm{d} R_{*}} \bar{F}_{\mathrm{D}}$

where we have taken

$\epsilon_{0} \equiv \frac{\Delta_{R}^{2}}{\Delta_{z}^{2}}$ and $\left(\frac{\psi_{\mathrm{N}}}{R_{0} \Delta_{R}}\right)^{2} \frac{1}{4 \pi \rho_{\mathrm{N}}}=\Omega_{0}^{2} \Delta_{z}^{2}$.

In addition, if we consider a configuration represented by

$\bar{\psi}_{*} \simeq\left(\sin R_{*}\right) \exp \left(-\frac{\epsilon}{2} R_{*}^{2}\right)$,

implying that

$\bar{\psi}^{\prime}=\left(\cos R_{*}-\epsilon R_{*} \sin R_{*}\right) \exp \left(-\frac{\epsilon}{2} R_{*}^{2}\right)$

and

$\bar{\psi}_{*}^{\prime \prime}=\left\{-\sin R_{*}+\sin R_{*}\left(\epsilon^{2} R_{*}^{2}-\epsilon\right)-2 \epsilon R_{*} \cos R_{*}\right\} \exp \left(-\frac{\epsilon}{2} R_{*}^{2}\right)$,

we have

$\bar{F}_{\mathrm{D}}=-\left\{1+\epsilon \sin ^{2} R_{*}\right\} \exp \left(-\epsilon R_{*}^{2}\right)$.

Then we rewrite Eq. (12.1) as

$\frac{\mathrm{d}}{\mathrm{d} R_{*}}\left(\bar{\rho}_{*}-\bar{F}_{\mathrm{D}}\right)+6 \epsilon_{0} R_{*}\left(\rho_{*}-\bar{F}_{\mathrm{D}}\right)=-6 \epsilon_{0} R_{*} \bar{F}_{\mathrm{D}}$

and define

$\mathrm{Y} \equiv \rho_{*}-\bar{F}_{\mathrm{D}}$, which gives

$\frac{\mathrm{d}}{\mathrm{d} R_{*}} \mathrm{Y}+6 \epsilon_{0} \mathrm{Y} R_{*}=-6 \epsilon_{0} R_{*} \bar{F}_{\mathrm{D}}$

To solve this equation we take

$\mathrm{Y}=H\left(R_{*}\right) \exp \left(-3 \epsilon_{0} R_{*}^{2}\right)$

and obtain

$\mathrm{e}^{-3 \epsilon_{0} R_{*}^{2}} \frac{\mathrm{d} H}{\mathrm{~d} R_{*}}=-6 \epsilon_{0} R_{*}\left\{1+\epsilon \sin ^{2} R_{*}\right\} \mathrm{e}^{-\epsilon R_{*}^{2}}$.

Thus

$\epsilon=3 \epsilon_{0}$

and

$H\left(R_{*}\right)=H_{0}+3 \epsilon_{0} R_{*}^{2}+18 \epsilon_{0}^{2}$

$\int_{0}^{R} R_{*} \mathrm{~d} R_{*} \sin ^{2} R_{*}$.

In conclusion we may choose to consider the "ring" density profile,

$$
\begin{aligned}
\bar{\rho}_{*}= & \left\{1+3 \epsilon_{0}\left[R_{*}^{2}+\sin ^{2} R_{*}+6 \epsilon_{0} \int_{0}^{R_{*}} \mathrm{~d} R_{*}\left(R_{*} \sin R_{*}\right)\right]\right\} \\
& \times \exp \left(-3 \epsilon_{0} R_{*}^{2}\right) .
\end{aligned}
$$

To identify the radial temperature profile that corresponds to the solution represented by Eqs. (12.3) and (12.13), we refer to Eq. (7.3) and analyze the function

$p_{* \mathrm{M}}\left(R_{*}\right)=-\left(\frac{\psi_{\mathrm{N}}}{4 \pi R_{0} \Delta_{R}}\right)^{2}\left\{\epsilon_{0} \bar{\psi}_{*}^{2}+\bar{\psi}_{*}+\bar{\psi}_{*} \frac{\mathrm{d}^{2} \bar{\psi}_{*}}{\mathrm{~d} R_{*}^{2}}\right\}$.

We note that

$\psi \psi^{\prime \prime}=\left\{-\sin ^{2} R_{*}+\sin R_{*}^{2}\left(\epsilon^{2} R_{*}^{2}-\epsilon\right) \sin R_{*}\right\} \exp \left(-\epsilon R_{*}^{2}\right)$.

Consequently,

$$
\begin{aligned}
p_{* \mathrm{M}}= & \left(\frac{\psi_{\mathrm{N}}}{4 \pi R_{0} \Delta_{R}}\right)^{2}\left\{\left(1+2 \epsilon_{0}-9 \epsilon_{0}^{2} R_{*}^{2}\right) \sin ^{2} R_{*}\right. \\
& \left.+6 \epsilon_{0} R_{*} \cos R_{*} \sin R_{*}\right\} \exp \left(-3 \epsilon_{0} R_{*}^{2}\right)
\end{aligned}
$$

and

$$
\begin{aligned}
\frac{T_{\mathrm{M} *}}{m_{i}}= & \frac{p_{* \mathrm{M}}}{\rho} \\
& \propto \frac{\left(1+2 \epsilon_{0}-9 \epsilon_{0}^{2} R_{*}^{2}\right) \sin ^{2} R_{*}+6 \epsilon_{0} R_{*} \cos R_{*} \sin R_{*}}{1+3 \epsilon_{0}\left[R_{*}^{2}+\sin ^{2} R_{*}+6 \epsilon_{0} \int_{0}^{R *} \mathrm{~d} R_{*}\left(R_{*} \sin ^{2} R_{*}\right)\right]} .
\end{aligned}
$$

Therefore we can verify that the total temperature $T_{\mathrm{G}}+T_{\mathrm{M}}$ can remain positive for all values of $R_{*}$. 


\section{Magneto-gravitational modes equation}

We may now argue that the class of configurations treated in the previous sections should emerge as the fully nonlinear saturated stage of the magneto-gravitational modes that we analyze in this section and in Sect. 14. These can be excited from a "conventional" disk configuration (Pringle 1981), and for their excitation they involve the product of the vertical gravitational force and the radial density gradient in addition to the differential rotation. One of these modes was identified in Coppi (2009) for a fairly extreme asymptotic limit. We note that, as in the case of the original magneto rotational instability (Velikhov 1959), the presence of seed (vertical) field, even if small, is essential for the onset of these modes.

The linearized equation that we use to describe magnetogravitational modes, for which both the differential rotation term $\rho \Omega_{\mathrm{D}}^{2}=-2 \rho \Omega R(\mathrm{~d} \Omega / \mathrm{d} R)$ and the gravitational term $z \Omega_{\mathrm{K}}^{2} \partial \rho / \partial R$ were of key importance, was derived in Coppi (2009). On the other hand, only a special class of solutions of this equation was considered. Therefore, without repeating the derivation we consider normal mode perturbations from the initial state, that is, a "conventional" currentless axisymmetric plasma disk, in which the gravity associated with the central object prevails, and the Keplerian velocity is the only one present. The relevant axisymmetric modes are represented by the perturbed toroidal velocity

$\hat{v}_{\phi}=\tilde{v}_{\phi}(z) \exp \left[\gamma_{0} t+\mathrm{i} k_{R}\left(R-R_{0}\right)\right]$

where $R_{0}$ is a radial distance at which the modes are considered to be localized and $k_{R}^{2} R_{0}^{2} \gg 1$. Moreover,

$\hat{v}_{R}=\gamma_{0} \hat{\xi}_{R}$ and $\hat{v}_{z}=\gamma_{0} \hat{\xi}_{z}$

are the other perturbed velocity components where, in particular, $\hat{\xi}_{z}=\tilde{\xi}_{z}(z) \exp \left[\gamma_{0} t+\mathrm{i} k_{R}\left(R-R_{0}\right)\right]$.

We indicate Fourier transform of $\tilde{\xi}_{z}(z)$ by $\tilde{\xi}(k)$, and the relevant dispersion equation is

$\left\{\left(\frac{7}{3}\right)^{2}\left(\frac{\gamma_{0}}{v_{\mathrm{A}}}\right)^{2}-k^{2}\left(\epsilon_{\mathrm{K}}-\frac{k^{2}}{k_{0}^{2}}\right)\right\} \tilde{\xi}(k) \simeq$

$\frac{1}{H_{0}^{2}}\left\{\frac{4 k_{0}^{2}}{15} D_{\mathrm{N}} \frac{\mathrm{d}^{2}}{\mathrm{~d} k^{2}} \tilde{\xi}+k \frac{\mathrm{d}^{2}}{\mathrm{~d} k^{2}}(k \tilde{\xi})\right\}$.

Here, modes that are vertically localized around $z=0$ over distances smaller than $H_{0}$, for equilibrium density profiles represented by $\rho \simeq \rho_{0}\left(1-z^{2} / H_{0}^{2}\right)$, are considered, and the local equilibrium temperature gradient is included in the definition of

$D_{\mathrm{N}} \equiv 1-\frac{3}{2} \eta_{T}$

where $\eta_{T}=\left(\mathrm{d} \ln T / \mathrm{d} z^{2}\right) /\left(\mathrm{d} \ln \rho / \mathrm{d} z^{2}\right)$. The temperature $T$ is defined by $p=2 n T$ and $n=\rho / m_{i}$ where $m_{i}$ is the ion mass. Clearly, the rhs of Eq. (13.1) vanishes if the local density and temperature gradients vanish. Moreover, $v_{\mathrm{A}}^{2}=B_{z}^{2} /\left(4 \pi \rho_{0}\right)$ where $B_{z}$ is the seed vertical field, $k_{0}^{2} \equiv \Omega_{\mathrm{D}}^{2} / v_{\mathrm{A}}^{2}, k_{R}^{2} \simeq k_{0}^{2}$ in the sense that $\epsilon_{\mathrm{K}}=1-k_{R}^{2} v_{\mathrm{A}}^{2} / \Omega_{\mathrm{D}}^{2} \ll 1$, and we assume $k_{0}^{2} H_{0}^{2} \gg 1$. This corresponds to assuming that $2 T / m_{i} \gg v_{\mathrm{A}}^{2}$.

\section{Magneto-gravitational modes profiles and growth rates}

Since it is convenient to deal with dimensionless variables we, define

$\bar{k}^{2} \equiv k^{2} L_{0}^{2}$ and $L_{0}^{2} \equiv\left(\frac{15}{4}\right)^{1 / 3} \frac{H_{0}^{2 / 3}}{k_{0}^{4 / 3}}$.
Then we take

$\mathcal{E}_{0} \equiv \epsilon_{\mathrm{K}}\left(\frac{15}{4}\right)^{1 / 3}\left(H_{0} k_{0}\right)^{2 / 3}$

and

$\Gamma_{0}^{2} \equiv\left(\frac{7}{3}\right)^{2}\left(\frac{15}{4}\right)^{2 / 3} \frac{H_{0}^{2 / 3}}{k_{0}^{4 / 3}}\left(\frac{\gamma_{0}}{v_{\mathrm{A}}}\right)^{2}$,

and the dispersion equation to be analyzed becomes

$\left\{\Gamma_{0}^{2}-\mathcal{E}_{0} \bar{k}^{2}+\bar{k}^{4}\right\} \tilde{\xi}=D_{\mathrm{N}} \frac{\mathrm{d}^{2} \tilde{\xi}}{\mathrm{d} \bar{k}^{2}}+\epsilon_{\mathrm{N}} \bar{k} \frac{\mathrm{d}^{2}}{\mathrm{~d} \bar{k}^{2}}(\bar{k} \tilde{\xi})$

for $\epsilon_{\mathrm{N}} \equiv(15 / 4)^{2 / 3} /\left(k_{0} H_{0}\right)^{2 / 3} \ll 1$.

Therefore if we consider rather flat or inverted temperature profiles, such that $D_{\mathrm{N}}>0$ and finite, we can find localized solutions around $\bar{k}_{m}= \pm\left(\mathcal{E}_{0} / 2\right)^{1 / 2}$, where $\mathrm{d} U / \mathrm{d} \bar{k}=0$ for $U \equiv \bar{k}^{4}-\mathcal{E}_{0} \bar{k}^{2}+\Gamma_{0}^{2}$. Around these points $U \simeq-\mathcal{E}_{0}^{2} / 4+\Gamma_{0}^{2}+$ $2 \mathcal{E}_{0}\left(\bar{k}-\bar{k}_{m}\right)^{2}$. Then we take $\mathcal{E}_{0}^{2} \gg 1, \Gamma_{0}^{2} \simeq \mathcal{E}_{0}^{2} / 4-\Delta \Gamma_{0}^{2}$ and $U \simeq-\Delta \Gamma_{0}^{2}+2 \mathcal{E}_{0}\left(\bar{k}-\bar{k}_{m}\right)^{2}$. In this case around $\bar{k}=\bar{k}_{m}$, Eq. (14.2) becomes

$D_{\mathrm{N}} \frac{\mathrm{d}^{2} \tilde{\xi}}{\mathrm{d} \bar{k}^{2}} \simeq\left[2 \mathcal{E}_{0}\left(\bar{k}-\bar{k}_{m}\right)^{2}-\Delta \Gamma_{0}^{2}\right] \tilde{\xi}$

and has the solution $\tilde{\xi}=\tilde{\xi}_{0} \exp \left[-\Sigma\left(\bar{k}-\bar{k}_{m}\right)^{2} / 2\right]$ where $\Sigma^{2} \equiv$ $\left(2 \mathcal{E}_{0} / D_{\mathrm{N}}\right)$ and $\Sigma=\Delta \Gamma_{0}^{2} / D_{\mathrm{N}}$. Consequently, we consider $2 \mathcal{E}_{0}>D_{\mathrm{N}}$.

In general, such as, in the case where $\mathcal{E}_{0} \sim 1$, the asymptotic solution of Eq. (14.2) takes the form

$\tilde{\xi} \simeq \frac{1+\Gamma_{0}^{2} \bar{k}^{2} /\left(2 D_{\mathrm{N}}\right)}{1+\mathcal{E}_{0} \bar{k}^{4} /\left(12 D_{\mathrm{N}}\right)}$

for $\bar{k} \ll 1$ and $\tilde{\xi} \propto \exp \left(-|\bar{k}|^{3}\right)$ for $\bar{k}^{2} \gg 1$. Thus it will have a minimum at $\bar{k}=0$ and two symmetric maxima before decaying for $\bar{k}^{2} \rightarrow 0$. To evaluate $\Gamma_{0}^{2}$ as a function of $\mathcal{E}_{0}$, we can make use of the quadratic form

$\mathcal{E}_{0}\left\langle\tilde{\xi}^{2} \bar{k}^{2}\right\rangle=\Gamma_{0}^{2}\left\langle\tilde{\xi}^{2}\right\rangle+\left\langle\bar{k}^{4} \tilde{\xi}^{2}\right\rangle+D_{\mathrm{N}}\left\langle\left(\frac{\mathrm{d} \tilde{\xi}}{\mathrm{d} \bar{k}}\right)^{2}\right\rangle$

where

\langle\rangle$=\int_{-\infty}^{+\infty} \mathrm{d} \bar{k}$

considered as a variational principle for $\mathcal{E}_{0}$ for given values of $\Gamma_{0}^{2}$. In particular, the instability threshold $\mathcal{E}_{0}^{s}$ can be evaluated from Eq. (14.5) by taking $\Gamma_{0} \equiv 0$.

If instead we refer to peaked temperature profiles such that

$D_{T} \equiv \frac{3}{2} \eta_{T}-1>0$,

we look for solutions to the equation

$\left\{\Gamma_{0}^{2}-\mathcal{E}_{0} \bar{k}^{2}+\bar{k}^{4}\right\} \tilde{\xi}=-D_{T} \frac{\mathrm{d}^{2} \tilde{\xi}}{\mathrm{d} \bar{k}^{2}}+\epsilon_{\mathrm{N}} \bar{k} \frac{\mathrm{d}^{2}}{\mathrm{~d} \bar{k}^{2}}(\bar{k} \tilde{\xi})$,

where $\epsilon_{\mathrm{N}} \ll 1$, which are localized near $\bar{k}=0$. These take the form $\tilde{\xi} \simeq \tilde{\xi}_{0} \exp \left(-\Sigma \bar{k}^{2} / 2\right)$ when $\Sigma^{2} \gg 1$, requiring that $D_{T} \Sigma^{2}=$ $\mathcal{E}_{0}$ and $D_{T} \Sigma=\Gamma_{0}^{2}$. Thus $\Gamma_{0}^{2}=\left(\mathcal{E}_{0} D_{T}\right)^{1 / 2}$, and we see that $\Gamma_{0}^{2}$ depends both on the temperature vertical gradient through $D_{T}$ 
and the differential rotation through $\mathcal{E}_{0}$. Clearly this asymptotic form of the solution of Eq. (14.7) is valid for $\mathcal{E}_{0} / D_{T} \gg 1$.

In general the relevant solution of Eq. (14.7) has a single maximum at $\bar{k}=0$ and is only marginally stable for $D_{T}=0$. In this case, the solution reported earlier in Coppi (2009) again takes of the form $\tilde{\xi} \simeq \tilde{\xi}_{0} \exp \left(-\Sigma \bar{k}^{2} / 2\right)$ but with $\Sigma^{2}=1 / \epsilon_{\mathrm{N}}, \Sigma=$ $\mathcal{E}_{0} /\left(3 \epsilon_{\mathrm{N}}\right)$ and $\mathcal{E}_{0}=3 \epsilon_{\mathrm{N}}^{1 / 2}$. Moreover, if we consider both $D_{T}$ and $\Gamma_{0}^{2}$ to be small, and in particular $\Gamma_{0}^{2} \sim \bar{k}^{4} \sim \epsilon_{\mathrm{N}}, \mathcal{E}_{0} \sim \bar{k}^{2}$, and $D_{T} \sim \epsilon_{\mathrm{N}} \bar{k}^{2}$, we have $D_{T} \sim \epsilon_{\mathrm{N}}^{3 / 2}, \quad \mathcal{E}_{0} \sim \epsilon_{\mathrm{N}}^{1 / 2}$, and $\Gamma_{0} \sim$ $D_{T}^{1 / 2} / \epsilon_{\mathrm{N}}^{1 / 4}$. More precisely, in this case $\tilde{\xi}$ takes the same form as in the case where $D_{T}=0$, with $\Sigma^{2}=1 / \epsilon_{\mathrm{N}}$ and $\Gamma_{0}^{2}=D_{T} \Sigma=$ $D_{T} / \epsilon_{\mathrm{N}}^{1 / 2}$ but $\mathcal{E}_{0}=3 \epsilon_{\mathrm{N}}^{1 / 2}+D_{T} / \epsilon_{\mathrm{N}}$. Then, in the limit where $\mathcal{E}_{0} \sim$ $D_{T} / \epsilon_{\mathrm{N}}$ we find $\Gamma_{0}^{2} \sim\left(\mathcal{E}_{0} D_{T}\right)^{1 / 2}$ and $\Sigma^{2} \sim \mathcal{E}_{0} D_{T}$ as expected.

\section{Tri-dimensional ring configurations}

Ring configurations have been found previously (Coppi 2009) on the basis of a linearized theory of tridimensional modes emerging from a "conventional" disk. In fact, these modes can be viewed as extensions of the linear modes discussed in the previous sections and can be represented by

$\hat{\xi}_{z} \simeq \tilde{\xi}_{z 0} \exp \left\{-\frac{\left(R-R_{0}\right)^{2}}{\Delta_{R}^{z}}-\frac{z^{2}}{2 \Delta_{z}^{2}}\right\}$
$\times \sin \left\{k_{R}\left(R-R_{0}\right)-m_{\phi}\left(\Omega_{0} t+\phi\right)\right\} \exp \left(\gamma_{0} t\right)$.

We can see that the dependence of $\hat{\xi}_{z}$ on $\left(R-R_{0}\right)$ is very similar to that of $\bar{\psi}_{*}\left(R_{*}\right)$ represented by Eq. (12.3). On the other hand $\Delta_{R} \simeq\left[\left(\gamma_{0} / \Omega_{0}\right) R_{0} /\left(k_{0} m_{\phi}\right)\right]^{1 / 2}$, where $m_{\phi}$ is the toroidal mode number, and clearly the finiteness of $\Delta_{R}$ depends on $m_{\phi}$ not being zero. In particular, the condition $k_{R} m_{\phi} \mathrm{d} \Omega_{\mathrm{k}} / \mathrm{d} R<0$ under which Eq. (15.1) is valid indicates that the relevant modes are trailing spirals.

When considering the configurations that can be identified by a nonlinear analysis, we may refer to the rings obtained in the rigid rotor limit (Sect. 12). Then we may take

$\boldsymbol{B}=\frac{1}{R}\left[\nabla \psi \times \boldsymbol{e}_{\phi}+\frac{\alpha_{\phi}}{\Delta_{z}} \psi \boldsymbol{e}_{\phi}\right]+\Delta \boldsymbol{B}_{\mathrm{p}}$

where $m_{\phi}$ is a relatively low number,

$\psi=\psi_{\mathrm{N}} \exp \left(-\frac{\bar{z}^{2}}{2}-R_{*}^{2}\right) \sin \left[R_{*}-m_{\phi}\left(\Omega_{0} t-\phi\right)\right]$

and

$$
\begin{aligned}
& \nabla \cdot\left(\Delta \boldsymbol{B}_{\mathrm{p}}\right)+\alpha_{\phi} \psi_{\mathrm{N}} \frac{m_{\phi}}{\Delta_{z} R^{2}} \cos \left[R_{*}-m_{\phi}\left(\Omega_{0} t-\phi\right)\right] \psi_{\mathrm{N}} \\
& \times \exp \left(-\frac{\bar{z}^{2}}{2}-R_{*}^{2}\right)=0 .
\end{aligned}
$$

Therefore $\left|\Delta \boldsymbol{B}_{\mathrm{p}}\right| /(|\Delta \psi| / R) \ll 1$ and, to lowest order in this ratio, the analysis given in Sect. 12 should remain valid. We note, however, that $\left.\boldsymbol{J} \times \boldsymbol{B})_{\phi}=\boldsymbol{J}_{\mathrm{p}} \times \Delta \boldsymbol{B}_{\mathrm{p}}\right)_{\phi} \neq 0$ in this case, where $\boldsymbol{J}_{\mathrm{p}}$ is the poloidal current. Thus as expected, a configuration represented by Eq. (15.2) involves a torque that will have to be compensated for in order to have a steady-state configuration to order $\left|\Delta \boldsymbol{B}_{\mathrm{p}}\right| /\left|B_{\mathrm{p}}\right|$.

\section{Theoretical outline for the generation of magnetic fields in the universe}

On the basis of the linearized theory of the modes that are excited in a disk configuration and of the nonlinear structures, in particular rings, that can evolve from disks as a result of the development of these modes, we may give the following outline for the production of significant magnetic field through the dynamics of plasmas surrounding compact astrophysical objects such as black holes.

a) Starting from a situation where no magnetic field is present, we may assume that nonthermal particle distributions, such as those with anisotropic pressures $\left(p_{\|} \neq p_{\perp}\right)$, are produced. These distributions can give rise to instabilities that can produce "seed" magnetic fields of limited amplitudes and relatively short wavelengths.

b) Assuming that these fields can interact to produce locally small fields with macroscopic wavelengths in the radial direction, magneto-gravitational modes can then be excited under the combined effects of gravity the vertical plasma pressure gradient, the differential plasma rotation, and other factors.

c) During the nonlinear evolution, the amplified magnetic field can become larger than the original seed field.

d) The saturated state of the instability can be represented by one of the stationary nonlinear configurations of the type that is described in particular in Sect. 12, and for which the magnetic energy density becomes comparable with the plasma thermal energy density (pressure).

Therefore we can propose a new theoretical line of analysis that can lead to an explanation of the generation of significant magnetic fields in the Universe. Clearly, the main steps of this line should be compared with the dynamo theories that preceded it.

\section{Concluding observations}

A theoretical framework to explain the generation of magnetic fields in the Universe has been given on the basis of two clear findings. One is that of magneto-gravitational modes that are driven by the combined effects of the product of the gravitational force, the plasma density gradients orthogonal to it, differential rotation, and other factors, when considering plasmas surrounding compact astrophysical objects such as black holes. The other is that of ring configurations by the relevant nonlinear analysis where the "driving factor" for the magnetic fields is the (vector) product of the gravitational force and the density gradient orthogonal to it. In the latter case, which can be envisioned as the result of the evolution of magneto-gravitational modes with a ring structure into the nonlinear regime, no trace of a "seed" magnetic field is left in the relevant nonlinear theory and the magnetic field pressure comparable to the gravitationally confined plasma pressure. As pointed out in the main body of the paper, there are gaps to be filled, the most important being the nonlinear evolution of the appropriate magneto-gravitational modes. The problem of identifying the simplest quasi stationary plasma configurations that can surround collapsed objects has led to limiting our attention to plasma regimes where the isorotation condition can be enforced, taking inspiration from the theory of equilibrium configurations developed for magnetically confined laboratory plasmas and extended to pulsar magnetospheres (Cohen et al. 1983).

In particular, the experimental observation of the socalled quasi periodic oscillations of X-ray emission, in one of the 
radiation emission regimes associated with galactic black holes (Remillard \& McClintock 2006), indicates that non axisymmetric configurations (Coppi \& Rebusco 2008) have to be included in the evolution of relevant plasma structures. We note that "solitary rings" have been found earlier in the linearized approximation when considering spiral structures that can emerge from simple disk configurations (Coppi 2009). Now we have shown that structures of the same kind are found in the nonlinear limit as well.

Referring to axisymmetric configurations, the conditions under which separable solutions in the variable $R-R_{0}$ and $z$ can be found (Sect. 7) deserve to be scrutinized. Therefore, an adequate (Regev \& Umurhan 2008) computational effort including the investigation of nonseparable solutions will have to be undertaken. Other issues to be dealt with are the connection between the fine- scale structure of the plasma configurations around black holes and the grand distribution of currents and electromagnetic fields on the larger scale around them (Blandford 1976; Lovelace 1976), the accretion flows, the appearance of jets in certain regimes, and relevant global transport processes such as angular momentum (Shakura \& Sunyaev 1973).

Moreover, we note that both the linear and nonlinear analyses that we reported here relied on the assumption that the particle distributions in momentum space are Maxwellian, so a scalar pressure can be defined. This is a serious limitation in view of the fact that there are important radiation regimes that have been observed experimentally (Remillard \& McClintock 2003), and are associated with compact objects, such as galactic black holes. They are definitely nonthermal. The case where a population with a strongly anisotropic pressure has therefore been analyzed recently. This was in addition to a thermal population, one with an isotropic pressure, which is smaller than that of the high energy population in the direction of the magnetic field. The main results, which will be reported in a later paper, are that the pressure anisotropy $\left(p_{\|}>p_{\perp}\right)$ makes a significant new contribution to the growth rate of magneto-gravitational modes and can produce a new kind of ring structures (Coppi 2012).

Acknowledgements. It is a pleasure to thank E. P. Velikhov for a series of stimulating conversations on magnetic field generation processes, A. Nekrasov for his timely and useful suggestions, T. Rabga for proposing Eq. (8.1) as a possible solution, P. Rebusco for her insightful questions, and T. Zhou for his work on the manuscript. The present work was sponsored in part by the US Department of Energy.

\section{Appendix A: Temperature and density vertical profiles for a "conventional" disk}

Given the importance that the parameter $\eta_{T}=$ $(\mathrm{d} \ln T / \mathrm{d} z) /(\mathrm{d} \ln \rho / \mathrm{d} z)$ has in the linearized theory of magneto-gravitational modes, we consider the vertical equilibrium equation

$\frac{\partial p}{\partial z}=-z \rho \Omega_{\mathrm{K}}^{2}-\frac{1}{8 \pi} \frac{\partial}{\partial z} B^{2}+\frac{1}{4 \pi}(\boldsymbol{B} \cdot \nabla \boldsymbol{B})_{z}$

that for $B=B_{z}$ reduces to

$\frac{\partial p}{\partial z^{2}}=-\frac{1}{2} \Omega_{\mathrm{K}}^{2} \rho=-\frac{1}{2} \Omega_{\mathrm{K}}^{2} m_{i} n\left(z^{2}\right)$,

where $p=2 n\left(z^{2}\right) T\left(z^{2}\right), \quad n=n_{0} \bar{n}\left(z_{*}^{2}\right), \quad T=T_{0} \bar{T}\left(z_{*}^{2}\right), \quad \bar{z}_{*}^{2}=$ $z^{2} / H_{\mathrm{G}}^{2}, \quad \bar{n}\left(z_{*}^{2}=0\right)=1, \quad \bar{T}\left(z_{*}^{2}=0\right)=1$, and

$H_{\mathrm{G}}^{2}=\frac{4 T_{0}}{m_{i} \Omega_{\mathrm{K}}^{2}}$.

Consequently,

$\frac{\mathrm{d} \bar{n}}{\mathrm{~d} z_{*}^{2}}=-\frac{\bar{n}}{\bar{T}}\left(1+\frac{\mathrm{d} \bar{T}}{\mathrm{~d} \bar{z}_{*}^{2}}\right)$

and

$\bar{n}=\exp \left[-\int_{0}^{z_{*}^{2}} \frac{\mathrm{d} z_{*}^{2}}{\bar{T}}\left(1+\frac{\mathrm{d} \bar{T}}{\mathrm{~d} \bar{z}_{*}^{2}}\right)\right]$.

In particular, if we take $\bar{T} \simeq 1-\alpha_{T} z_{*}^{2}$, we have $\bar{n} \simeq 1-\left(1-\alpha_{T}\right) z_{*}^{2}$, $\eta_{T} \simeq \alpha_{T} /\left(1-\alpha_{T}\right)$ and $H_{0}^{2} \simeq H_{\mathrm{G}}^{2} /\left(1-\alpha_{T}\right)$.

\section{References}

Bertin, G. 2000, Dynamics of Galaxies (Cambridge University Press) Blandford, R. D. 1976, MNRAS, 176, 465

Cohen, R., Coppi, B., \& Treves, A. 1973, ApJ, 179, 269

Coppi, B. 2009, A\&A, 504, 321

Coppi, B. 2011, Phys. Plasmas, 18, 032901

Coppi, B. 2012a, Invited Lecture for the Astrophysical and Space Plasmas International School (I.S.S.S. L'Aquilia, Italy), http://www.cifs-isss . org/scientific-program. asp

Coppi, B., \& Rebusco, P. 2008, E.P.S. Conference on Plasma Physics, Crete, Greece, Paper P5.154

http://epsppd.epfl.ch/Hersonissos/html/c_index.htm

Coppi, B., \& Rousseau, F. 2006, ApJ, 641, 458

Remillard, R. A., \& McClintock, J. E. 2006, ARA\&A, 44, 49

Lovelace, R. V. E. 1976, Nature, 262, 649

Pringle, J. E. 1981, ARA\&A, 19, 137

Regev, O., \& Umurhan, O. H. 2008, A\&A, 481, 1

Shakura, N. I., \& Sunyaev, R. A. 1973, A\&A, 24, 337

Velikhov, E. P. 1959, Sov. Phys. JETP, 36, 995 